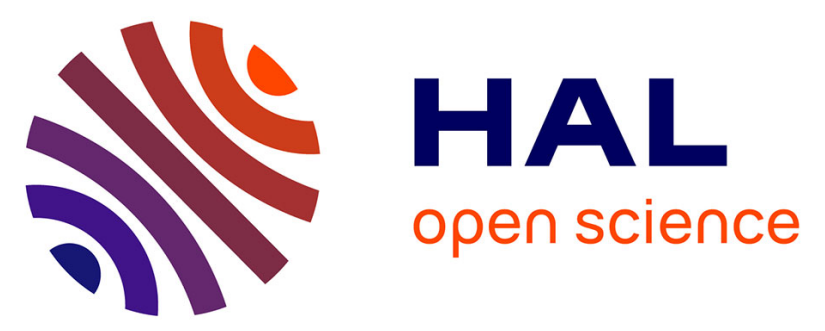

\title{
Large surface gamma cameras for medical imaging: characterization of the bismuth germanate blocks
}

M. Fontana, D. Dauvergne, R. Della Negra, J.M. Létang, F. Mounier, É.

Testa, L. Zanetti, Y. Zoccarato

\section{- To cite this version:}

M. Fontana, D. Dauvergne, R. Della Negra, J.M. Létang, F. Mounier, et al.. Large surface gamma cameras for medical imaging: characterization of the bismuth germanate blocks. Journal of Instrumentation, 2018, 13, pp.P08018. 10.1088/1748-0221/13/08/P08018 . hal-01871818

\section{HAL Id: hal-01871818 \\ https://hal.science/hal-01871818}

Submitted on 28 Mar 2019

HAL is a multi-disciplinary open access archive for the deposit and dissemination of scientific research documents, whether they are published or not. The documents may come from teaching and research institutions in France or abroad, or from public or private research centers.
L'archive ouverte pluridisciplinaire HAL, est destinée au dépôt et à la diffusion de documents scientifiques de niveau recherche, publiés ou non, émanant des établissements d'enseignement et de recherche français ou étrangers, des laboratoires publics ou privés. 


\title{
Large surface gamma cameras for medical imaging: characterization of the bismuth germanate blocks
}

\author{
M. Fontana, ${ }^{a}$ D. Dauvergne, ${ }^{b}$ R. Della Negra, ${ }^{a}$ J. M. Létang, ${ }^{c}$ F. Mounier, ${ }^{a}$ É. Testa,${ }^{a}$ L. \\ Zanetti, $^{a}$ Y. Zoccarato ${ }^{a}$ on behalf of the CLaRyS collaboration \\ ${ }^{a}$ University of Lyon, Université Claude Bernard Lyon 1, CNRS-IN2P3, Institut de Physique Nucléaire de \\ Lyon, F-69622, Villeurbanne, France \\ ${ }^{b}$ LPSC, Université Grenoble-Alpes, CNRS/IN2P3 UMR 5821, Grenoble, France \\ ${ }^{c}$ University of Lyon, INSA-Lyon, Université Claude Bernard Lyon 1, UJM-Saint Étienne, CNRS, Inserm, \\ Centre Léon Bérard, CREATIS UMR 5220 U1206, F-69373, Lyon, France \\ E-mail: m. fontana@ipnl.in2p3.fr
}

\begin{abstract}
The CLaRyS collaboration focuses on the development of gamma detectors for medical applications, in particular for what concerns the range monitoring in ion beam therapy. Part of the research program aims to implement two gamma-camera clinical prototypes, a multi-collimated camera and a Compton camera. A common absorber detector has been designed for the two prototypes, based on bismuth germanate (BGO) blocks, $3.5 \times 3.8 \times 3 \mathrm{~cm}^{3}$, assembled in various geometrical configurations to meet the application requirements. The surface of each block is streaked in a matrix of $8 \times 8$ pseudo pixels, which makes possible a position reconstruction via Anger logic from the signals collected by four read-out photo-multiplier tubes. The whole set of available blocks comes from a dismantled positron emission tomography system by Siemens, so that each single block must be tested and characterized in terms of space, time and energy response. We present in this work the implemented characterization method, which leads to a complete estimation of the block response via gamma source irradiations and data analysis devoted to a custom calibration for the imaging performance optimization of each detector module. A reference set of blocks has been completely characterized and showed very homogeneous responses: the average energy resolution is $25 \%$ FWHM at $511 \mathrm{keV}$ and $20 \% \mathrm{FWHM}$ at $1275 \mathrm{keV}$, the time resolution ranges between 3.9 and $5.3 \mathrm{~ns}$ FWHM and the spatial resolution has been verified to be limited to the pseudo-pixel size.
\end{abstract}

KEYwORDS: Compton imaging, Gamma camera, Instrumentation for hadron therapy 


\section{Contents}

1 Introduction 1

2 Material and methods $\quad 2$

2.1 Acquisition systems 4

2.2 Space and energy calibration and characterization 5

$\begin{array}{lll}2.2 .1 & \text { Calibration process } & 6\end{array}$

2.2.2 Qualitative test of spatial reconstruction accuracy 6

$\begin{array}{lll}\text { 2.2.3 Pixel identification and energy calibration algorithm } & 6\end{array}$

2.3 Time response characterization 8

3 Results $\quad \mathbf{1 0}$

3.1 PMT gain equalization $\quad 10$

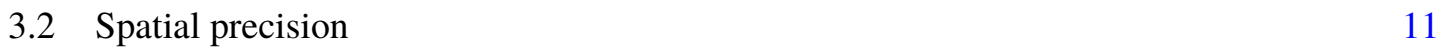

$\begin{array}{lll}3.3 & \text { Pixel identification } & 12\end{array}$

$\begin{array}{ll}3.4 & \text { Pixel energy calibration } \\ 3.5 & 13\end{array}$

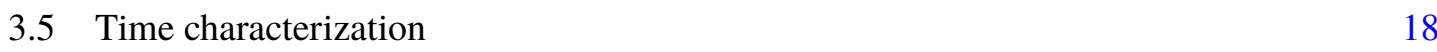

$\begin{array}{lll}3.6 & \text { Results for a set of blocks } & 18\end{array}$

4 Discussion and conclusions $\quad 18$

\section{Introduction}

The real-time monitoring of particle therapy treatment delivery is one of the main improvement needed to fully profit from the advantageous characteristics of this tumor irradiation method $[1,2]$. The dose delivered to the volume to be treated should be checked on-line in order to detect severe deviations from the treatment planning. The possibility to perform on-line monitoring would allow for a reduction of the safety margins currently applied in clinical practice and for a complete exploitation of the favorable depth-dose profile of hadrons in matter [3]. Several techniques have been proposed and explored for this purpose, and, among them, the measurement of the emitted secondary prompt-gamma rays is promising thanks to the very short emission time (within a nanosecond after the beam interaction) and the verified correlation between depth-dose beam profile and prompt-gamma emission profile $[4,5]$. The CLaRyS collaboration, involving four French research institutions, is tackling this monitoring issue with the parallel development of two prompt-gamma detection systems, a multi-collimated camera and a Compton camera. The Compton camera prototype (described in [6]) is composed of two detector sections: a scatterer, where the incoming photons are expected to be scattered via Compton interaction, and an absorber, where the scattered photons are ideally fully absorbed, since prompt-gammas are poly-energetic. The emission cone is reconstructed via analytical or iterative algorithms based on Compton kinematics. 
It requires the spatial and energy deposited information collected by the two detector sections; the overlap of all the reconstructed cones allows one to retrieve the prompt-gamma emission profile. The scatterer provides what is usually defined as "electronic collimation", but a mechanical collimation is still an option: a collimated camera can be obtained by removing the scatterer section and setting a physical collimator. With the latter configuration, the parallel incoming photons can traverse the collimator slits (which can be designed with different configurations depending on the desired application) and reach the absorber, where a full absorption is not necessarily required. Energy selections can be applied to discard gamma rays not correlated to the range. The spatial information relies then on the collimator geometry and the consequent selection of incoming gammas.

The CLaRyS prototypes share the components of the absorber detector, bismuth germanate (BGO) blocks from a dismantled Siemens HR+ PET system, similar to the ones first introduced in the middle of 80's for PET application [7]. The block design and performance were optimized for PET applications, so that for the detection of $511 \mathrm{keV}$ photons in a ring geometry. The employed detectors, readout by photo-multiplier tubes, are being tested for the new gamma detection system, which must be able to deal with photons in the prompt-gamma energy range, i.e. from about $100 \mathrm{keV}$ to approximately $10 \mathrm{MeV}$. Moreover, the block and photo-multiplier responses are being characterized in terms of space, energy and time accuracy.

We describe in this work the method used for the BGO block characterization and calibration, which relies on the work presented in [8] and [9] and on the calibration process described in [10], and is here extended with more refined features. The method is illustrated for one reference block, and the obtained results are shown for a set of tested blocks.

\section{Material and methods}

Bismuth germanate ( $\mathrm{BGO}-\mathrm{Bi}_{4} \mathrm{Ge}_{3} \mathrm{O}_{12}$ ) is one of the most used scintillators for gamma detection applications, thanks to a fair energy resolution and an optimal gamma detection efficiency, given the high effective atomic number (75) and high density $\left(7.12 \mathrm{~g} / \mathrm{cm}^{3}\right)$ [11] which maximize the photo-electric effect [12]. Moreover, the absence of internal radioactivity which characterizes other scintillator materials employed in the same field (e.g. LYSO), makes it suitable for low noise detectors, required by a Compton camera to reduce the amount of random coincidences, one of the main sources of background for the application in ion beam therapy monitoring [13].

Thirty BGO blocks have been recovered from a dismantled positron emission tomography system, and are now available to compose the absorber detector of the two gamma-camera prototypes under development. Each block has a surface of $3.5 \times 3.8 \mathrm{~cm}^{2}$, with a thickness of $3.0 \mathrm{~cm}$. The mono-block BGO crystal is streaked in a $8 \times 8$ pseudo-pixel matrix; a reflecting material is inserted between the pseudo-pixels to improve the light collection and optimize the spatial information accuracy via pixel separation. The read-out is achieved via four photo-multiplier tubes (PMTs) per block, composing a quartet, coupled to the block back surface. Thanks to the internal streaked structure of the block, the scintillation light is shared on the four PMTs depending on the pseudopixel where the interaction takes place (in case of multiple interactions more than one pseudo-pixel can be involved). The streaks have a different thickness according to their position in the block: they fully cover the block thickness on the block borders and they progressively shorten towards the block center, with a mono-block structure in the central block section on the entrance face. The 
reconstruction of the position of interaction is done via Anger logic, i.e. with a center of gravity calculation. The lateral surfaces of the block, as well as the half of the PMT length, are covered by a reflecting material which ensures the complete collection of the scintillation light. The whole structure (except the entrance window) is then protected by a $1 \mathrm{~mm}$ thick aluminum foil, which also isolates from external light contamination.

A custom front-end card has been designed and produced by the Laboratoire de Physique de Clermont-Ferrand (LPC) research group AVIRM and is used for the read-out of each BGO block. The card is equipped with four voltage modulators which divide the provided high voltage on the four photo-multiplier tubes. The voltage sent to each PMT can be tuned by means of potentiometers on these modules.

Figure 1 shows one reference block with the related front-end card, the component description is detailed in the figure caption. Figure 2 displays both a picture and a scheme of the absorber configuration with its mechanical support.

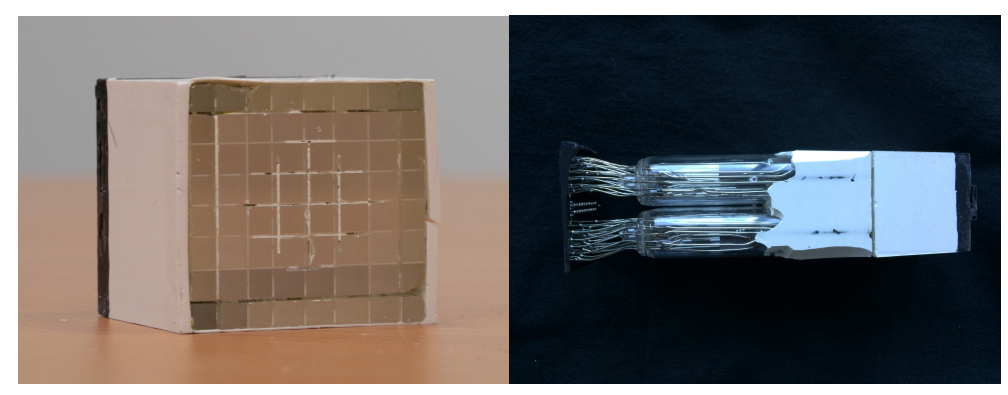

(a)

(b)

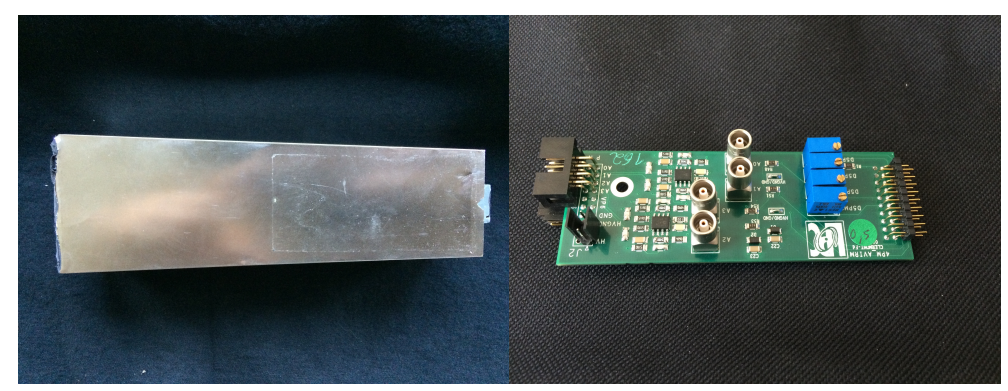

(c)

(d)

Figure 1: Figure 1a shows one BGO block before the coupling to the PMT quartet. The streaked structure is clearly visible, as well as the white reflecting material separating the pseudo-pixels and the one surrounding the block lateral sides. The same material also covers part of the photomultiplier tubes, as shown in picture 1b, where the four PMTs are glued to the block back surface. The whole detector is shielded by the aluminum cover in figure 1c, while figure $1 \mathrm{~d}$ shows the front-end card, with the blue modulator modules for the PMTs high voltage and the four analogical outputs used to retrieve the raw signals. 


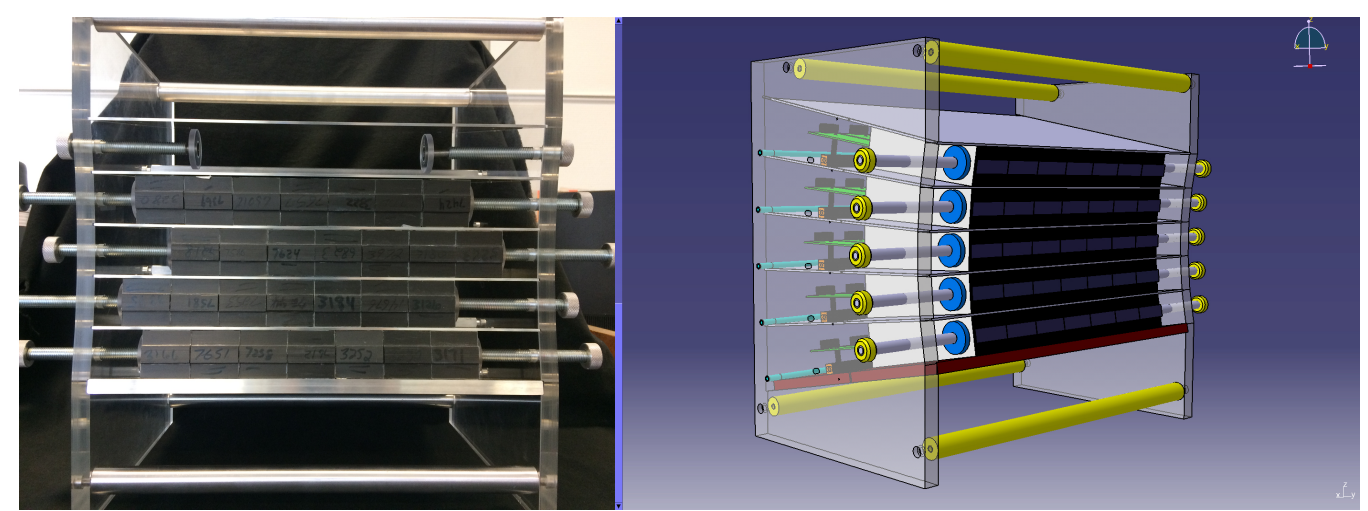

Figure 2: Absorber front view with the BGO block lines arranged in the mechanical support (left). Scheme of the BGO absorber with its mechanical support (right).

\subsection{Acquisition systems}

The signals produced by the four PMTs of each block are collected via four analog outputs on the front-end card (see figure 1d). The four retrieved signals per event are treated via standard NIM modules in order to be adapted to the acquisition systems. In particular, leading edge discriminators were used for logic signal processing.

Two different acquisition systems have been used for this characterization work. First, a National Instruments PXIe equipped with two flash ADC read-out modules, eight channels each, is used for the spatial and energy characterization and calibration of the tested blocks. The raw signals coming from the four PMTs are amplified and shaped via NIM modules (quad shaping bipolar amplifiers), which were fine-tuned via a pulse generator in order to adapt the amplification factor of each channel (an amplification factor of about 50 has been applied to the raw signals). The amplified signals are then split in order to be treated for trigger purpose. The trigger is based on the sum of the four signals, and a fixed threshold is applied for background rejection. The employed discriminator provides the logical trigger signal, which is sent to the trigger input of the ADC modules on the PXI. The four amplified signals, conveniently delayed, are sent as inputs to the ADC modules on the PXI, together with the sum signal which is used for experimental verification of the acquisition setup. A LabVIEW-based acquisition software, developed for this particular application at Institut de Physique Nucléaire de Lyon (IPNL), provides real time event visualization together with a partial, on-line spatial reconstruction of the events, and stores them in text files for further analysis. A second threshold can be set at the software level in case particular selections are needed during the acquisition, otherwise the event selection is performed at the analysis stage. A scheme of the acquisition setup for the spatial and energy calibration is given in figure 3 .

Concerning the timing characterization measurements, an eight-channel signal digitizer at 3.2 GS/s has been employed for high time resolution measurements. The so-called WaveCatcher has been developed by the Laboratoire de l'Accélérateur Linéaire (LAL) in Orsay and the Institut de Recherche sur les lois Fondamentales de l'Univers (IRFU) in CEA-Saclay, and its features are detailed in [14]. The digitizer is connected to the acquisition PC via USB port, and the data read out and storage are performed thanks to a custom acquisition software. The measurements are 


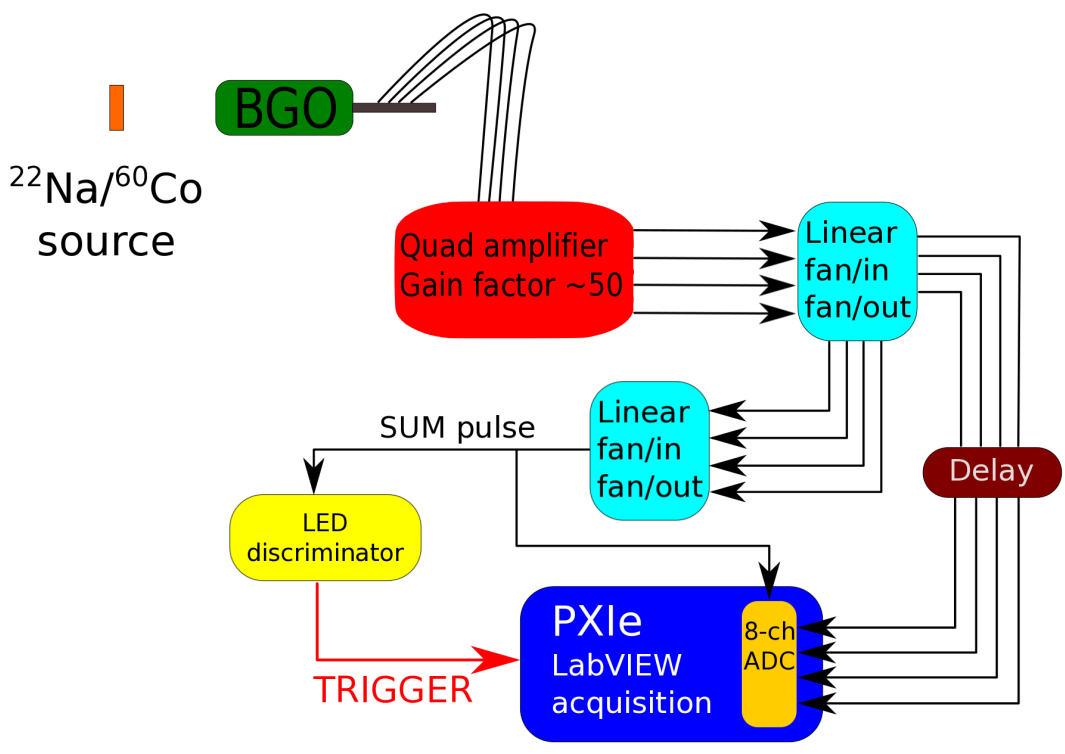

Figure 3: Scheme of the spatial and energy characterization test-bench.

based on the coincidence detection of back-to-back $511 \mathrm{keV}$ photons emitted by a ${ }^{22} \mathrm{Na}$ radioactive source, in order to be able to compare the time response of the tested BGO block to a reference scintillator with a fast and known response. An external trigger is then provided to the WaveCatcher by treating the BGO and reference scintillator signals with logic coincidence NIM modules, after proper discrimination. Further details are given in section 2.3, together with a scheme of the acquisition setup (figure 5).

\subsection{Space and energy calibration and characterization}

Each detector module must be characterized in terms of spatial and energy response, and the readout PMTs have to be calibrated to obtain a uniform response on the whole block surface. The characterization is performed with the irradiation by gamma sources, emitting photons at defined energies: in particular, we used $511 \mathrm{keV}$ and $1275 \mathrm{keV}$ photons from a ${ }^{22} \mathrm{Na}$ source, and the two photons emitted by a ${ }^{60} \mathrm{Co}$ source, at energies of $1173 \mathrm{keV}$ and $1332 \mathrm{keV}$.

The ${ }^{22} \mathrm{Na}$ source is a cylindrical source with an active diameter of $4 \mathrm{~mm}$, and an activity of about $400 \mathrm{kBq}$ : it has been placed at a distance of about $5 \mathrm{~cm}$ from the block entrance surface, with the center of the source facing the center of the block transverse surface. The ${ }^{60} \mathrm{Co}$ source has been installed in a lead cylindrical container ( $12 \mathrm{~cm}$ radius and $35 \mathrm{~cm}$ height), equipped with three different apertures: point-like $\left(2 \times 2 \mathrm{~mm}^{2}\right)$, linear $\left(2 \times 50 \mathrm{~mm}^{2}\right)$ and squared $\left(50 \times 50 \mathrm{~mm}^{2}\right)$. The activity of this ${ }^{60} \mathrm{Co}$ source is about $1.7 \mathrm{MBq}$, and the square shape has been used to obtain an homogeneous irradiation of the BGO block, with the block set with the center of the entrance surface corresponding to the source position, at a distance of $12 \mathrm{~cm}$. 


\subsubsection{Calibration process}

The space and energy calibration process is mainly divided into three stages:

- PMT gain equalization $\left({ }^{22} \mathrm{Na}\right)$ : the block is irradiated with the ${ }^{22} \mathrm{Na}$ source and the raw ADC distributions for the four PMTs are retrieved. The upper-energy falloff in the raw ADC spectra are taken as reference to equalize the distributions. Four energy calibration factors are extracted and used to the data correction; this correction corresponds to the PMT gain equalization.

- Pixel identification $\left({ }^{60} \mathrm{Co}\right)$ : once the calibration factors are extracted thanks to the ${ }^{22} \mathrm{Na}$ irradiation data, the block is exposed to the ${ }^{60} \mathrm{Co}$ source. The collected data are analyzed as in the previous step and calibrated according to the already calculated correction factors. The energy spectrum, the mono-dimensional spatial projections and the flood map are produced. The custom algorithm briefly described in the following section 2.2 .3 has been developed to identify the pseudo-pixel positions on the flood map. It is applied to the ${ }^{60} \mathrm{Co}$ irradiation data and the pseudo-pixel position map is stored.

- Pixel energy calibration: the ${ }^{22} \mathrm{Na}$ irradiation data are re-analyzed in this last calibration step in order to assign each interaction to a single pseudo-pixel according to the pixel position map obtained with the ${ }^{60} \mathrm{Co}$ data. Then the energy spectrum of each pixel can be produced and the two identified peaks (corresponding to the two photon energies emitted by the ${ }^{22} \mathrm{Na}$ source: $511 \mathrm{keV}$ and $1275 \mathrm{keV}$ ) can be used to equalize the pseudo-pixel response. The sum of all the pixels spectra produces the block energy spectrum. At this stage, the ADC channel values are calibrated to obtain the absorbed energy values in $\mathrm{keV}$.

It is worth noticing that the ${ }^{60} \mathrm{Co}$ irradiation is useful for the pixel identification given the high energy and narrow energy range of the emitted photons. The equalization factors obtained with the ${ }^{22} \mathrm{Na}$ source irradiation have been verified to be consistent with the ${ }^{60} \mathrm{Co}$ irradiation data, as expected. The whole method results to be robust.

\subsubsection{Qualitative test of spatial reconstruction accuracy}

Specific acquisitions have been devoted to the test of the block spatial accuracy potential. The detector is expected to be able to locate the collected interaction on the pseudo-pixel grid, but the possible sub-pixel accuracy can be tested with a collimated source scan of the block surface. The ${ }^{60} \mathrm{Co}$ source has been employed for this purpose, with the linear aperture. The block has been placed on a moving table with no distance between its entrance surface and the collimator aperture. The whole surface has been irradiated with horizontal and vertical movements of $1 \mathrm{~mm}$ and $2 \mathrm{~mm}$ per step. The collected data have been calibrated with the factors obtained with the homogeneous ${ }^{22} \mathrm{Na}$ irradiation, as explained in the previous section. We present and qualitatively discuss the results in section 3.2.

\subsubsection{Pixel identification and energy calibration algorithm}

Subsequent to that gain equalization process, an automatic iterative algorithm has been developed to identify the block pseudo-pixel positions. Integrated mono-dimensional spatial projections are 
computed along rows and columns of the calibrated flood map in order to derive the peak and valley positions in the distributions with the ROOT [15] methods included in the TSpectrum class and simple analytical calculations. With the resulting valley positions, a first rectangular grid of pseudopixels is initialized. The projection of each identified pseudo-pixel row (respectively column) is re-analyzed using the same integrated 1-D spatial projections along columns (respectively rows) looking for peaks and valleys as before. A refined grid is then produced, distorted from the initial rectangular one, and this process is iterated until a complete $8 \times 8$ pseudo-pixel grid is obtained.

Once the pseudo-pixels grid is fixed, the maximum of each pseudo-pixel region is automatically identified with a bin per bin scan in the defined area and its position defines the pseudo-pixel center relative position in the map.

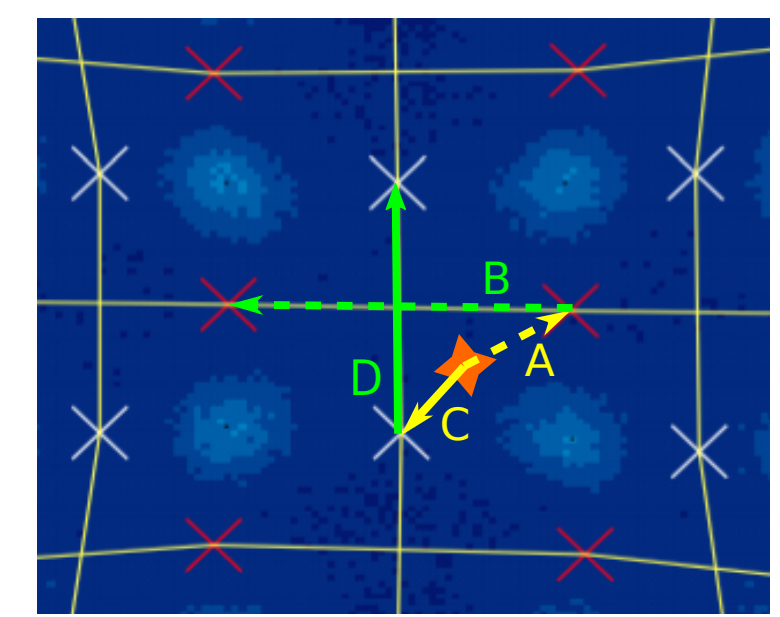

\section{$\succ$ Reconstructed interaction position}

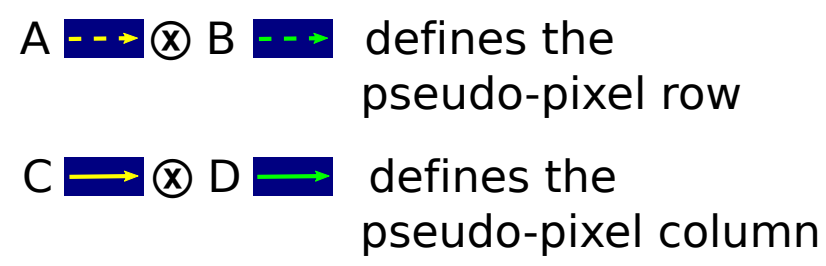

Figure 4: Logic for the event assignment to a single pixel. The dual pseudo-pixel grid is displayed: in white the mid points on the pseudo-pixel edges along rows (middle of lines in vertical direction), in red along columns (middle of lines in horizontal directions). The sign of the outer products detailed in the legend are used to define the column and row (so that the pseudo-pixel) where to assign the reconstructed event.

The event data are then assigned to the pseudo-pixels with the application of the following process, illustrated in Figure 4. The dual pseudo-pixel grid is created rows- and columns-wise to get the mid points on the pseudo-pixel edges. The even data assignment is then done by calculating the minimal distance between a column and a row average point with respect to the reconstructed event and then calculating two outer products, between the vector connecting the reconstructed point and 
closest column (row) average point and the vector connecting this average point to the previous or next one on the same column (row). The sign of the products defines the column (row) where to assign the interaction. Knowing the relative position of the interaction point with respect to the two minimal distance points on row and column, the correct pseudo-pixel is identified. A more simple approach could have been used, using the search of the minimal distance between the reconstructed event position and the pseudo-pixels centers, but it proved to be more liable to some assignment discrepancies due to the grid distortions. These distortions are mainly caused by the detection principle that relies on the pseudo-pixel separation via reflecting material, and can be corrected in event post-treatment with the a-priori geometrical knowledge of the block pseudo-pixel structure.

\subsection{Time response characterization}

The test-bench for the time response characterization has been set as shown in figure 5. $\mathrm{A} \mathrm{BaF}_{2}$ mono-block scintillator, read-out by a single photo-multiplier tube, has been used as reference detector. The reference scintillator and the BGO block under test have been set at arbitrary distances from the ${ }^{22} \mathrm{Na}$ source. The four raw signals coming out from the BGO block are summed

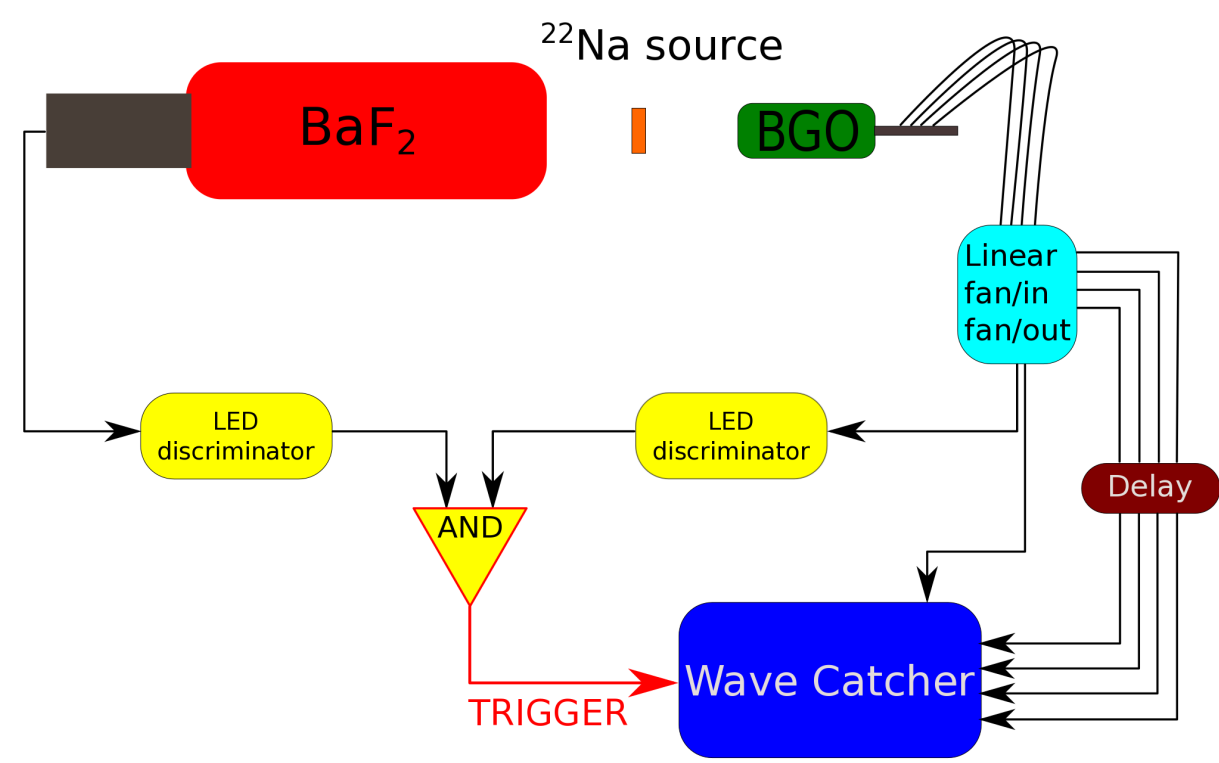

Figure 5: Scheme of the time response characterization test-bench.

with a linear Fan-in/Fan-out module, and the resulting signal is sent to a leading edge discriminator and converted to a logic signal according to a fixed threshold. The single signal emerging from the reference detector passes through a selected threshold and is converted to a logic signal. The two logic pulses, $100 \mathrm{~ns}$ width, are then sent to a coincidence module to create the trigger input for the WaveCatcher acquisition system described in section 2.1. The four BGO output raw signals, the reference scintillator raw output signal and the sum of the four raw BGO signals are sent to the WaveCatcher for digitization. An example of the collected raw signals from the studied BGO block is shown in figure 6: the four output signals of each single PMT are shown together with the 
resulting sum signal. The four signals exhibit a noisy feature that could be observed using the fast sampling acquisition, with a $80-100 \mathrm{MHz}$ oscillatory behaviour. At the present stage we did not infer the sources of this noise, that would be canceled by appropriate lower frequency filtering.

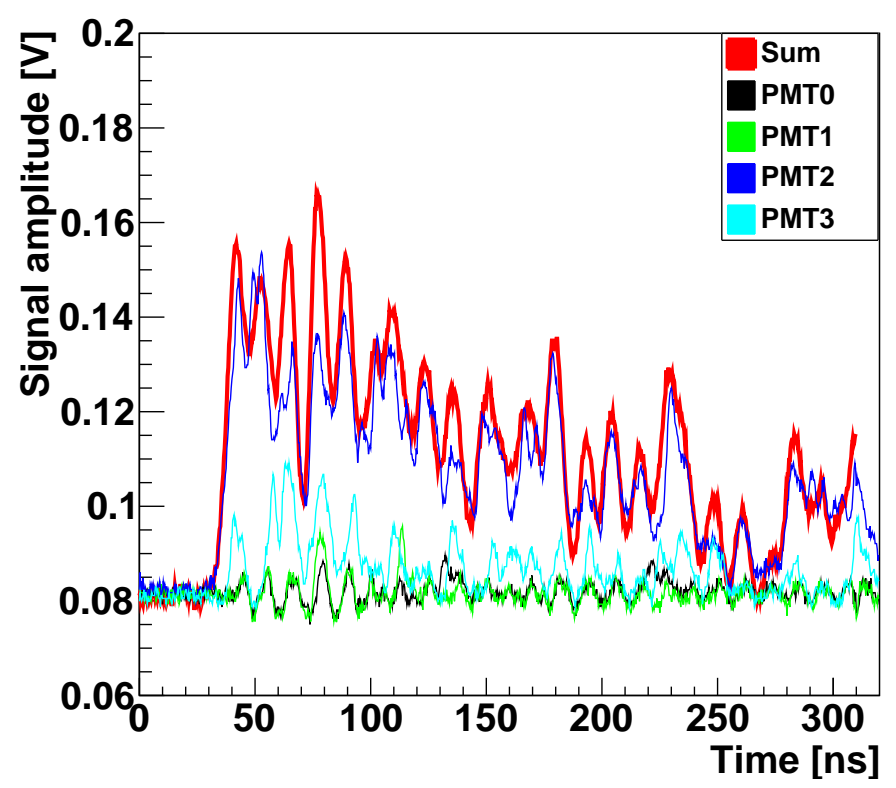

Figure 6: Reference BGO block raw signals collected with the Wave Catcher acquisition system. The four outputs of the single PMTs are shown together with the sum signal.

For each coincidence event, the six collected signals are analyzed with focus on the signal rising edge. The time corresponding to the amplitude maximum and to $20 \%, 30 \%, 50 \%$ and $80 \%$ of the maximum is retrieved for constant fraction discrimination tests. In addition to this, a fixed threshold is used for fixed value discrimination.

Different comparison methods have been tested in order to identify the more robust one for the definition of the time resolution of the blocks. The noisy structure of the single BGO raw signals (see figure 6) leads to very variable results depending on the defined threshold, and the more stable results are given by the comparison of the sum of the four BGO signals and the reference scintillator with the arrival time identified by a fixed tuned threshold. With this method, the arrival time of each signal can be defined and the time difference distribution of the two signals can be produced. The same analysis has been applied to a data set obtained with two identical $\mathrm{BaF}_{2}$ detectors exposed to the ${ }^{22} \mathrm{Na}$ source in coincidence. This data set allowed for the definition of the reference scintillator time resolution.

The resulting BGO time resolution is defined as the FWHM of arrival time difference distribution between BGO block and reference scintillator, with the subtraction of the reference scintillator contribution via uncertainties composition calculation. 


\section{Results}

\subsection{PMT gain equalization}

Figures 7 and 8 show the effect of the PMT gain equalization on the ADC count spectra and on the integrated mono-dimensional spatial distributions, while figures 9 and 10 show the effect of the PMT gain equalization on the block energy spectrum and two-dimensional spatial event distribution. All these results refer to the data collected with the reference BGO block irradiated with the ${ }^{22} \mathrm{Na}$ source.

In particular, Figures $7 \mathrm{a}$ and $7 \mathrm{~b}$ show the raw and equalized $\mathrm{ADC}$ profiles of the four read-out photo-multipliers (the peaks above $3000 \mathrm{ADC}$ counts in figure 7a correspond to the saturation of the NIM linear fan-in/fan-out module used to handle the data read-out; these values are rejected during the equalization stage). Figures $8 \mathrm{a}$ and $8 \mathrm{~b}$ show the projection on the two axes of the position of interaction reconstructed via Anger logic, before (left) and after (right) the PMT gain equalization. Figures $9 a$ and $9 b$ show the ADC event spectrum, obtained by the sum of the ADC values of the four PMTs, before (left) and after (right) the PMT gain equalization. Figures 10a and 10b show the two dimensional event position map (this will be called "flood map" in the following), before (left) and after (right) the PMT gain equalization; the interaction position is reconstructed via Anger logic.

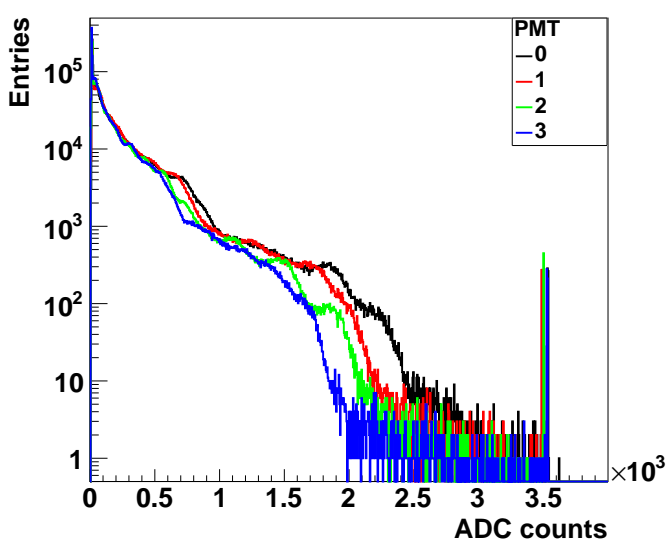

(a)

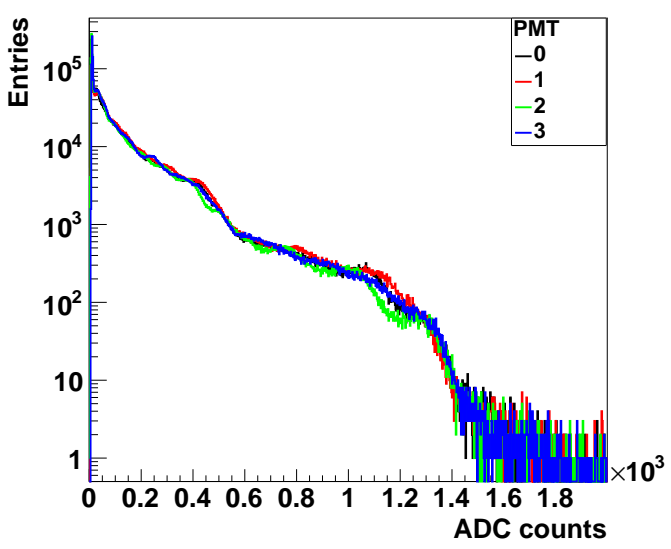

(b)

Figure 7: PMT signal amplitude spectra before (a) and after (b) the PMT gain equalization.

As shown by figures 7 to 10, the PMT gain equalization performed in this first calibration step is mandatory to optimize the spatial and energy response of the tested block. Figure $8 \mathrm{~b}$ highlights the better definition of the pseudo-pixels ensured by the gain equalization: the peak-to-valley ratio is increased, in particular for the most external pixels. The spatial response improvement is also reflected in a better energy response (from about $46 \%$ to $35 \%$ at $511 \mathrm{keV}$ and from approximately $40 \%$ to $30 \%$ at $1275 \mathrm{keV}$ ), as shown in figure $9 \mathrm{~b}$, where the two energy peaks of the ${ }^{22} \mathrm{Na}$ source are more narrow with respect to the ones obtained with the raw data. The flood map in figure 10b shows how the gain equalization and the offset tuning allow to arrange the position map over the whole block surface; the borders are better defined and the pseudo-pixels on the block limits (especially on the corner) are better separated. 

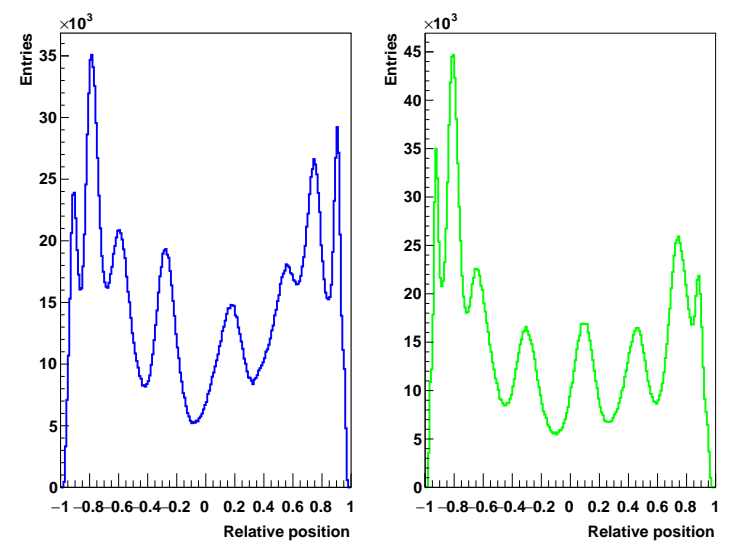

(a)
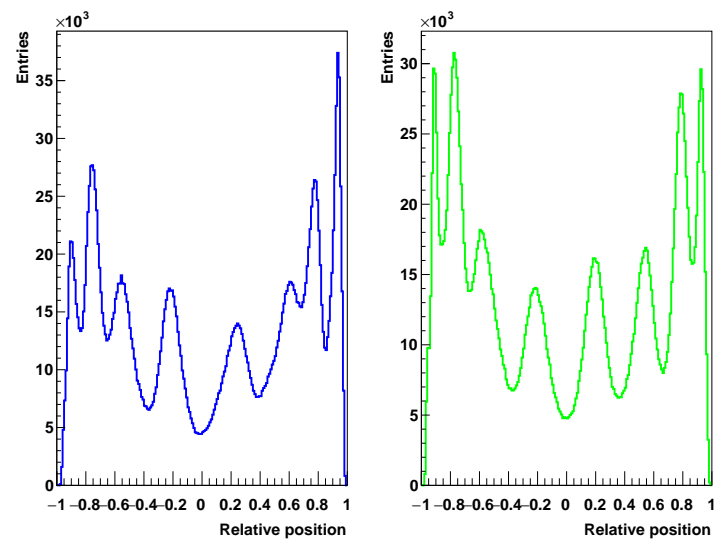

(b)

Figure 8: 1D integrated position distribution on the two transverse dimensions before (a) and after (b) the PMT gain equalization. In the two sub-figures, left side for the horizontal dimension, right side for the vertical one.

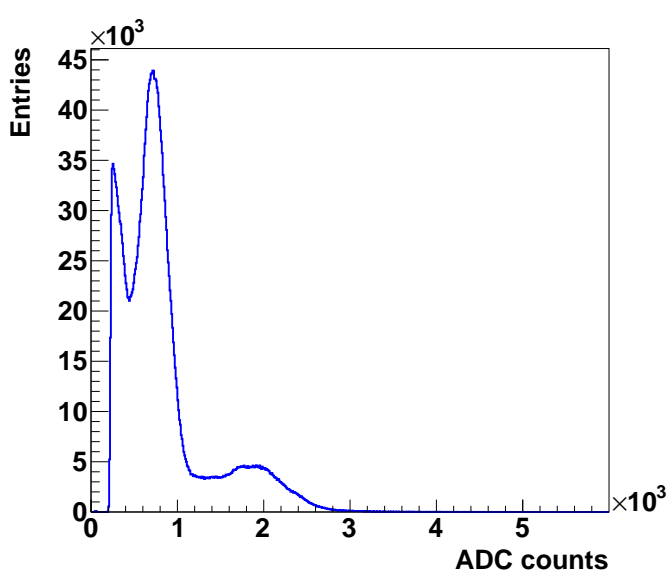

(a)

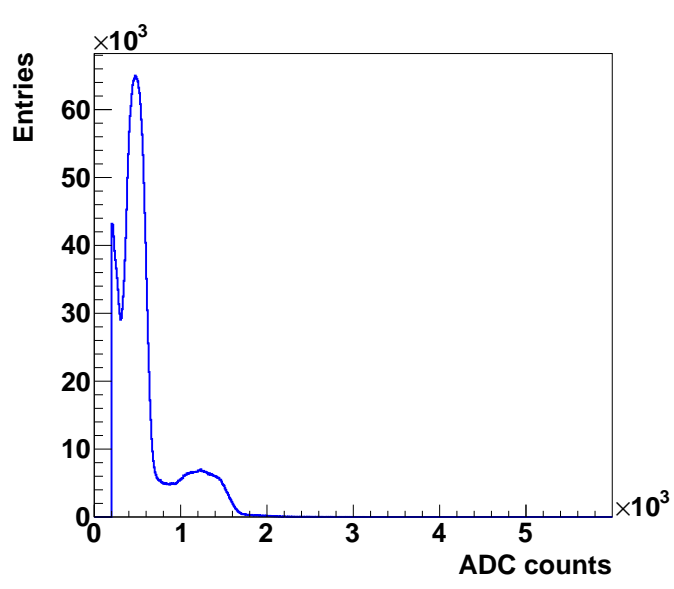

(b)

Figure 9: Block energy spectrum before (a) and after (b) the PMT gain equalization.

\subsection{Spatial precision}

Figure 11 shows four consecutive steps of the ${ }^{60}$ Co source collimated irradiation, with a $2 \mathrm{~mm}$ step movement from left to right and the linear aperture $\left(2 \times 50 \mathrm{~mm}^{2}\right)$ set in vertical position. A qualitative analysis is enough to appreciate the block spatial accuracy limitation. The presented four points of the scan irradiated a total of three pseudo-pixel columns, moving from the left geometrical border of one column to the center of the next one. As shown by figure $11 \mathrm{~b}$ and $11 \mathrm{~d}$, as the collimator aperture position is in front of the center of a pixel column, the whole pseudo-pixel surface is reconstructed: no difference is observed with respect to the same column in figure 10b, obtained with the homogeneous irradiation. In case the aperture faces the border between two pixel 


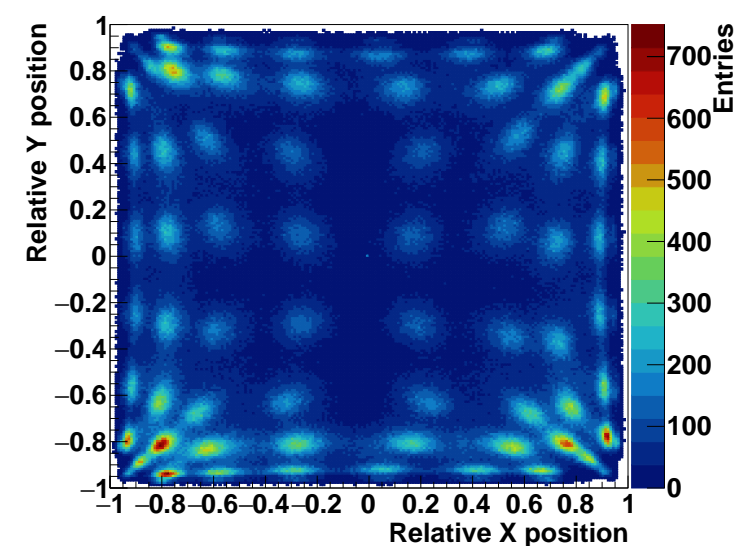

(a)

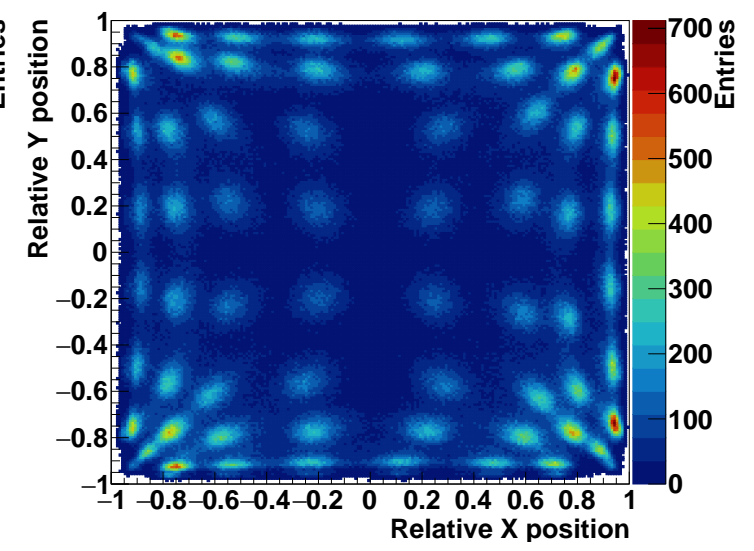

(b)

Figure 10: 2D reconstructed position map before (a) and after (b) the PMT gain equalization.

columns (figure 11a and 11c), the events are shared between the two. This could show a possibility of sub-pixel resolution for integrated measurements, which is anyway not reproducible on a single event basis. The same behavior has been verified with a vertical scan; the collimator and table configurations has been keep unchanged, while the block has been turned of $90^{\circ}$. To be noticed that the slit position reported in the presented figures is the geometrical one, while the two-dimensional maps represent the response of the tested block: the spatial reconstruction distortions lead to the mismatch with respect to the real position, which will be corrected at the data treatment stage.

In order to further confirm these results, a scan with reduced step size $(1 \mathrm{~mm})$ has been performed with the same collimator aperture, and no modifications have been detected in the spatial distribution of events for the steps centered on the same pseudo-pixel column. As before, when the collimator aperture faces the limit between two pseudo-pixels columns, the events are equally distributed on the two.

The 2D position maps of the vertical $2 \mathrm{~mm}$ step scan and of the $1 \mathrm{~mm}$ scan are not shown for conciseness; all these set of data verify that the spatial reconstruction accuracy of the employed blocks is limited to the pseudo-pixel size, and a sub-pixel resolution is not achievable on a single event basis.

\subsection{Pixel identification}

The results of the pixel identification algorithm described in section 2.2.3 are shown in figure 12 . Figure 12a shows the identified average values of the pseudo-pixels positions on the two transverse dimensions. As already detailed in the description section, starting from these average positions, the single pseudo-pixels positions in rows and columns are extracted and the "valleys" between neighboring pseudo-pixels are used to define the grid shown in figure $12 \mathrm{~b}$ together with the pseudopixel center position map. 


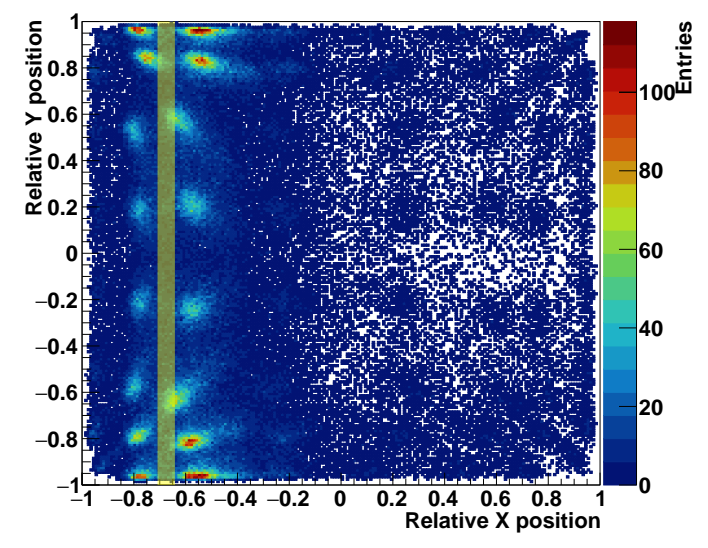

(a) Aperture center relative $\mathrm{X}$ position: -0.675

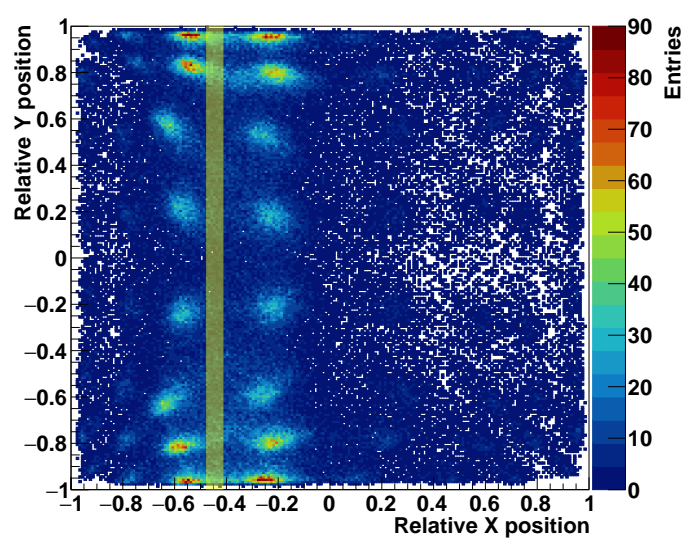

(c) Aperture center relative $\mathrm{X}$ position: -0.445

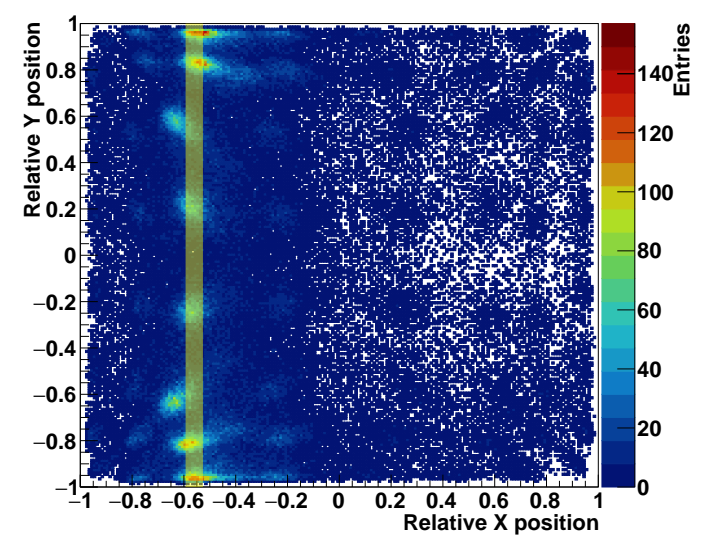

(b) Aperture center relative $\mathrm{X}$ position: -0.560

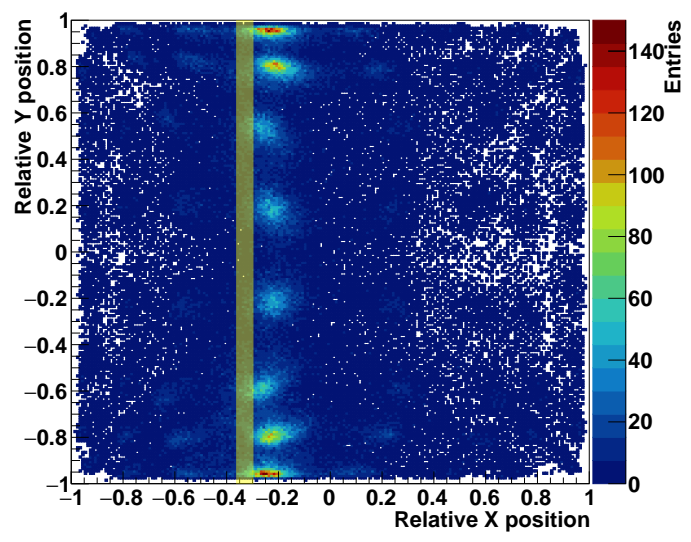

(d) Aperture center relative $\mathrm{X}$ position: -0.330

Figure 11: 2D reconstructed position maps during a 2-mm step scan performed with the ${ }^{60} \mathrm{Co}$ source with the line aperture. 4 reference measurements points, the position of the aperture is shown by the semi-transparent yellow band.

\subsection{Pixel energy calibration}

Once the pseudo-pixel positions and the related grid are defined, each interaction can be assigned to a single pixel. The energy spectrum of each pixel is then separately studied in order to equalize the energy response on a pixel basis.

The assignation method described in section 2.2.3 makes use of the dual pseudo-pixel position map shown in figure 13. The position assignement scheme to pseudo-pixels is shown in figure 14: a color has been given to each reconstructed point according to the pseudo-pixel region where it has been assigned by the described method. The method robustness is verified by the comparison of this map to the grid in figure $12 \mathrm{~b}$ and 13 .

In figure 15 the results of the pseudo-pixel energy calibration are shown. Figure 15a (left) shows the overlap of the energy spectra for 60 pseudo-pixels before the energy calibration and 

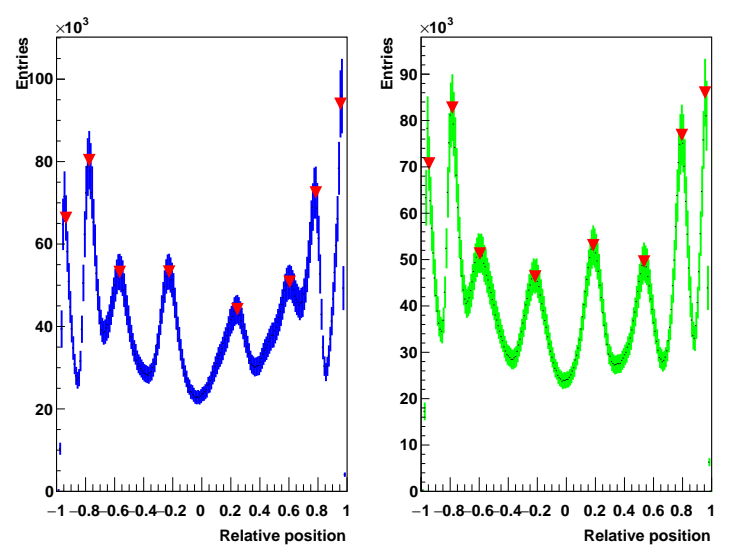

(a)

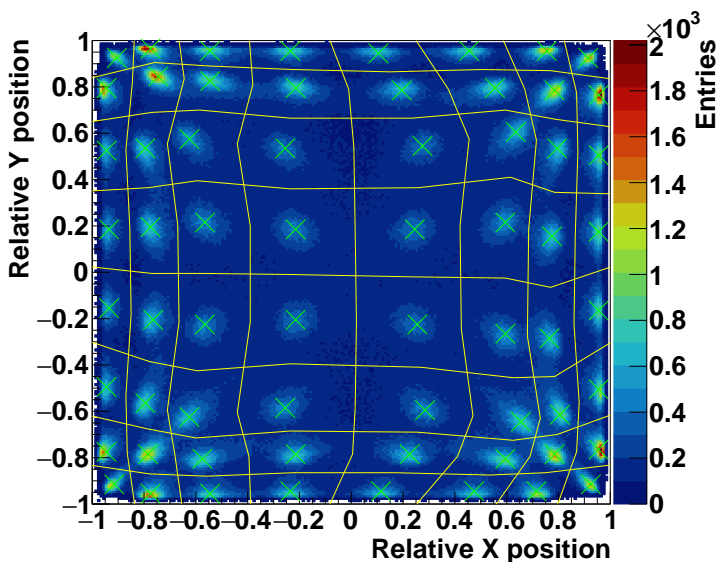

(b)

Figure 12: 1D integrated position distributions on the two transverse dimensions with the retrieved position of the pseudo-pixel average center (a). Reconstructed 2D map with the identified pseudopixels positions and surfaces.

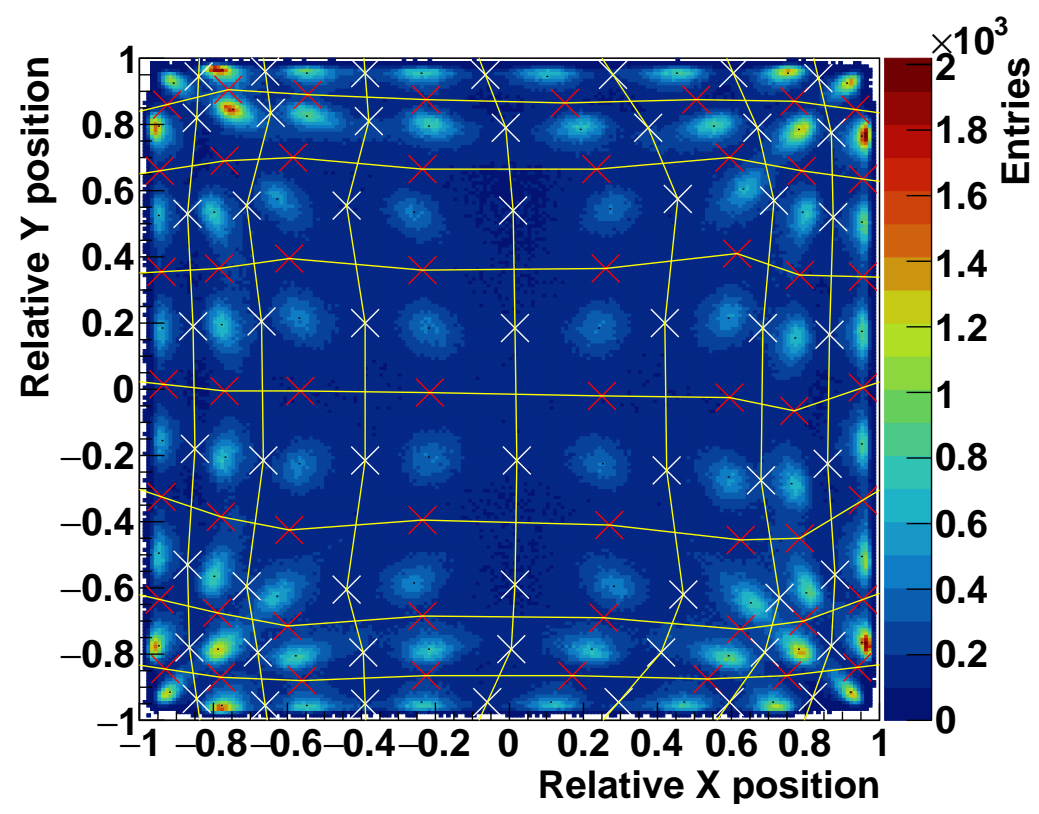

Figure 13: Dual pseudo-pixel position map used for the assignment of the reconstructed events to a single pixel. The highlighted points represent the valleys between neighboring pixels in the two directions.

equalization. The four pseudo-pixels on the corners show a very different energy response and are not included in this picture. This is probably due to a not complete light collection. The different response of each pixel to the two energy peaks is clearly visible. For each spectrum the low energy peak is assigned to $511 \mathrm{keV}$, while the high energy one to $1275 \mathrm{keV}$. In this way, the spectra are 


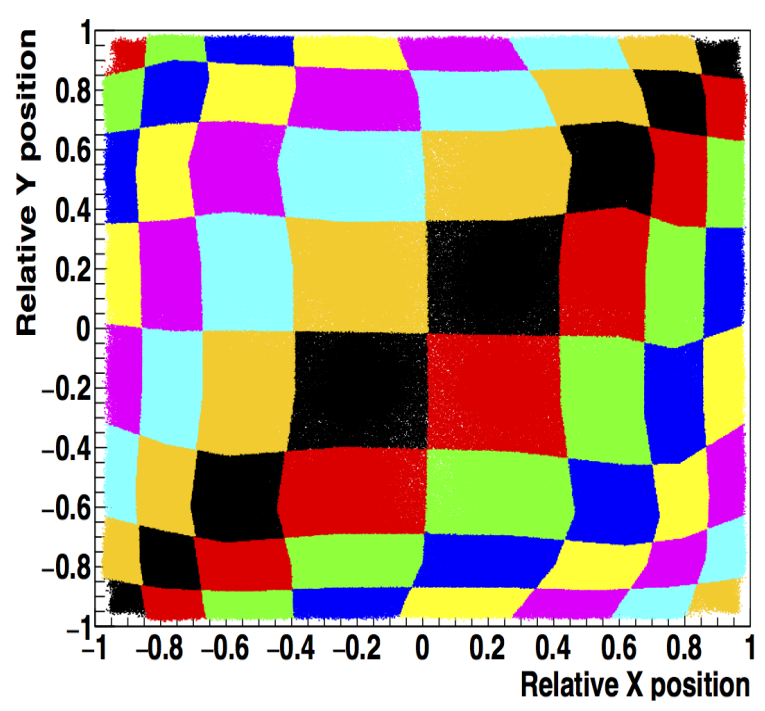

Figure 14: 2D map of the resulting interaction assignment: each event is colored according to the chosen pseudo-pixel.

linearly calibrated and equalized, as shown in figure 15a (right). For the four corner pseudo-pixels, the calibration is based only on the $1275 \mathrm{keV}$ peak, while the $511 \mathrm{keV}$ reference peak is substituted by the null ADC value.

Once the single pixel energy responses are equalized and calibrated, the whole block energy spectrum can be derived with the sum of the all pixels. In figure 15b (left) the ADC spectrum is shown before the equalization process, while in figure $15 \mathrm{~b}$ (right) the calibrated energy spectrum is presented. In figure $15 \mathrm{~b}$ (left) the energy spectra related to three reference position on the block surface are reported: this makes possible to appreciate the different contributions to the noncalibrated spectrum and the behavior of different block sections. The block peripheral pixels show an overall lower light collection and a lower efficiency in detecting high energy photons (reduced peak integral with respect to the center and mid-center pseudo-pixels). Concerning the central pixels, the slight inhomogeneity is probably due to the streaked structure which guides less than on the borders the scintillation photons. This aspect is further discussed in the following, as a comment to the results in figure 16.

The two spectra are represented in logarithmic scale in order to better appreciate the calibration effect: it allows for the optimization of the energy response on the two spectroscopic lines of the ${ }^{22} \mathrm{Na}$ source. At this stage, the energy resolutions of the block can be defined as the FWHM of the two energy peaks. In table 1, the energy resolutions derived after the calibration process are compared to the raw ones, obtained by the non-calibrated data. The results reported in table 1 show the need for the implemented calibration process, which allows to optimize the BGO block spatial and energy response.

Thanks to the assignment of each reconstructed event to a single pseudo-pixel, the relative efficiency can be evaluated on a single pseudo-pixel basis. The two color maps in figure 16 show the number of events collected by the 64 pseudo-pixels during the ${ }^{22} \mathrm{Na}$ source homogeneous 

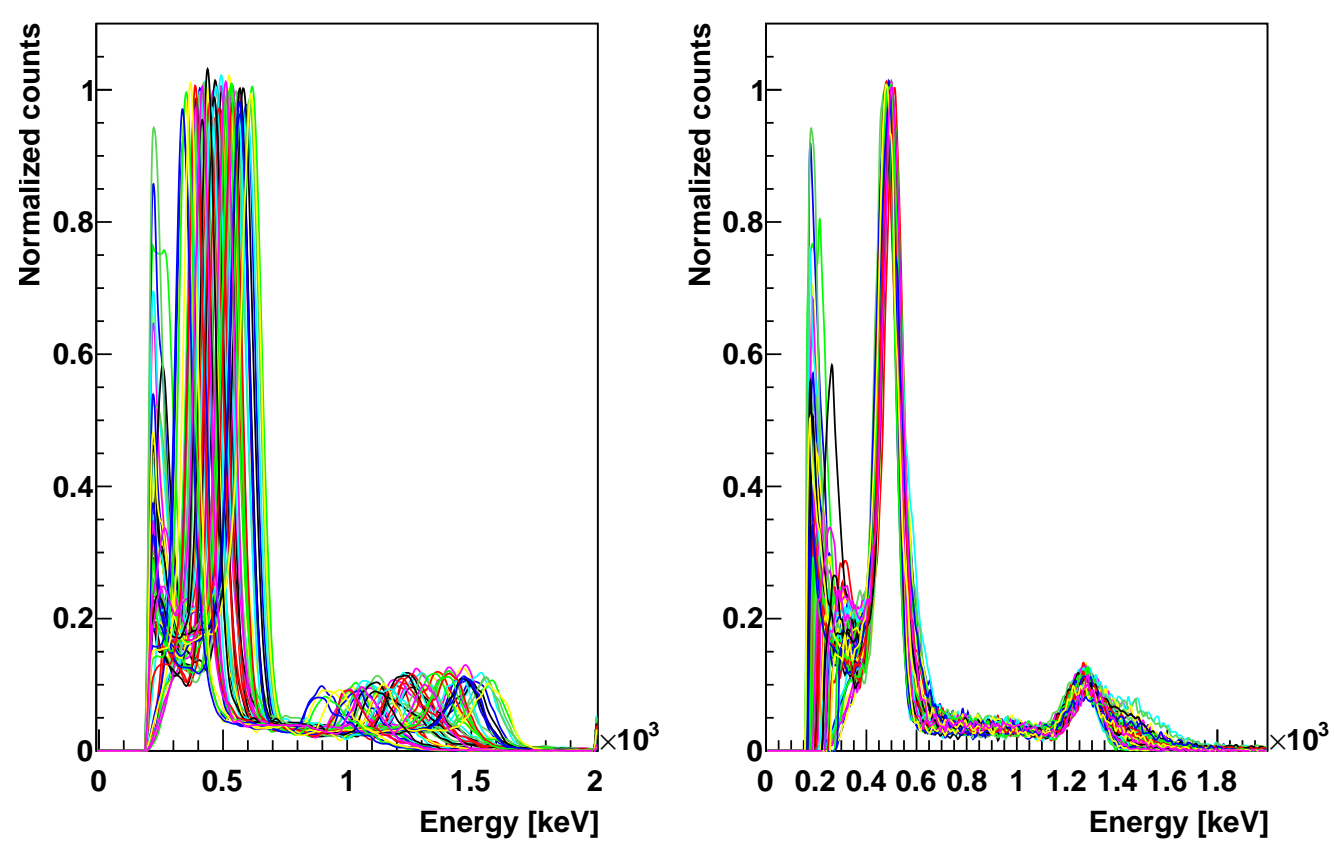

(a)
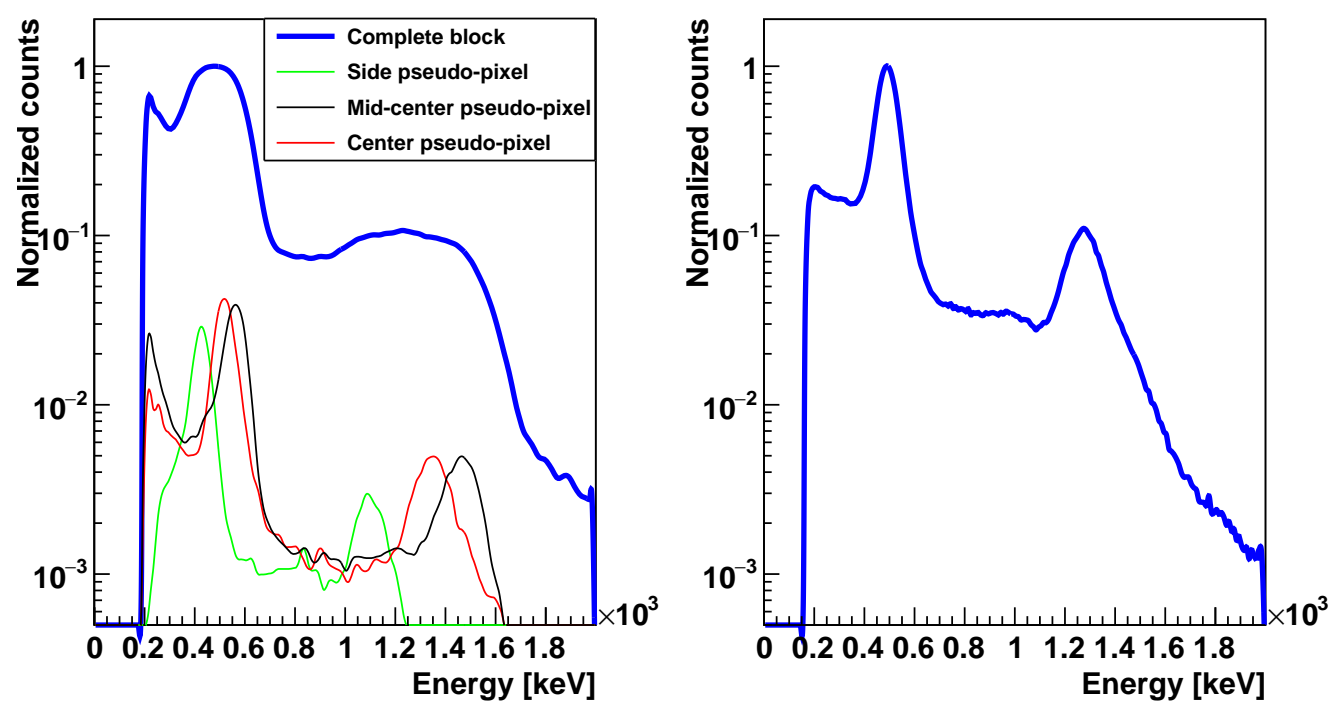

(b)

Figure 15: Single pseudo-pixels (a) and whole block (b) energy spectra with the ${ }^{22} \mathrm{Na}$ before (left) and after (right) the calibration process. The whole block spectra are reported in logarithmic scale. Three non calibrated spectra of pixels in reference positions (border, mid-center and center area) on the block are also reported with the non calibrated spectrum (a normalization factor has been applied for visualization purpose). 
Table 1: Comparison of the block energy resolutions at the two reference energies of $511 \mathrm{keV}$ and $1275 \mathrm{keV}$ before and after the calibration process.

\begin{tabular}{lcc}
\hline & $\begin{array}{c}\text { Energy resolution } \\
\text { @ 511 keV } \\
\text { FWHM [\%] }\end{array}$ & $\begin{array}{c}\text { Energy resolution } \\
\text { @ 1275 keV } \\
\text { FWHM [\%] }\end{array}$ \\
\hline Before equalization & 46.12 & 39.43 \\
\hline After equalization & 23.03 & 18.04 \\
\hline
\end{tabular}

irradiation, with an energy selection performed on the two photons energy emitted by the source (511 keV events in figure 16a and $1275 \mathrm{keV}$ events in figure 16b). The entries are normalized to the maximum number of entries in a pseudo-pixel, detected for a $511 \mathrm{keV}$ energy selection.

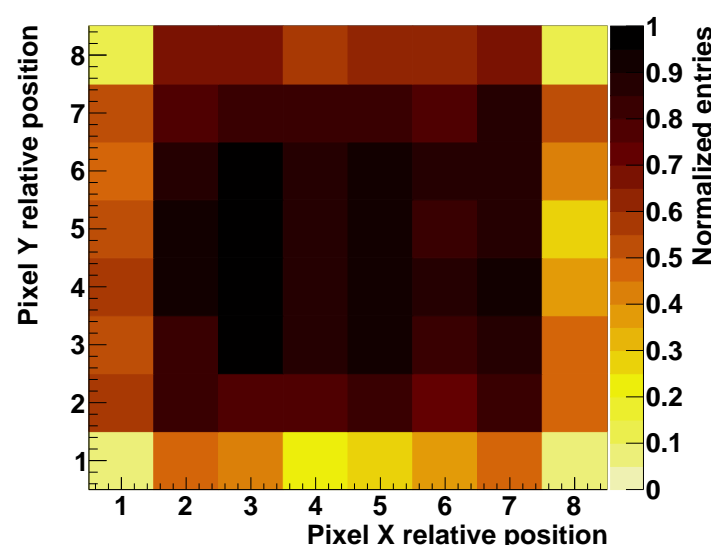

(a)

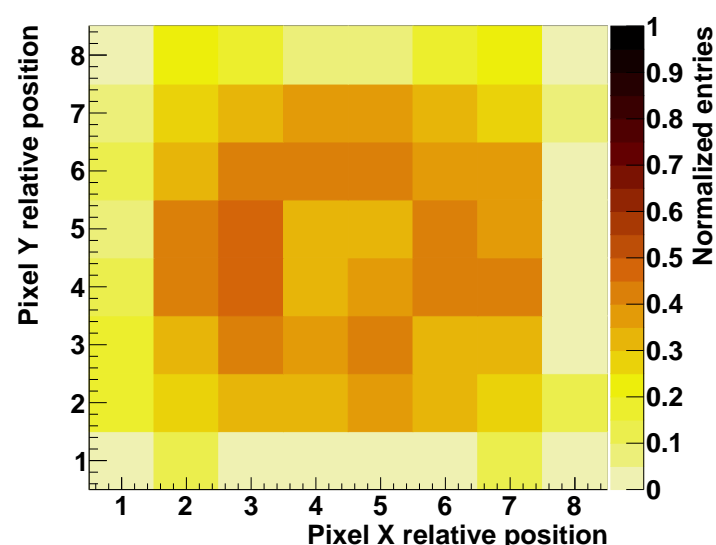

(b)

Figure 16: Relative number of entries for each pseudo-pixel as a function of the pixel relative position, represented by the row and column numbers ( 0 to 8 from left to right and bottom to top of the block surface). Figure (a) shows the entries in a selected energy window around $511 \mathrm{keV}$, figure (b) in an energy window around $1275 \mathrm{keV}$. All the entries are normalized to the maximum collected number of entries, corresponding to $511 \mathrm{keV}$ events in the central section of the block.

Figure 16 shows that the expected homogeneous distribution of events over the whole block surface is confirmed for the central pseudo-pixels of central lines ( 2 to 7 ), while the block borders present a factor 2-3 lower detection efficiency. In particular, the pseudo-pixels on the 4 corners (line 1 and 8, pseudo-pixels 1 and 8), have an efficiency of a factor between 5 and 6 lower with respect to the center of the block surface. This effect is partially due to geometrical factors, given the fact that the side pseudo-pixels are slightly smaller with respect to the central one (as also shown by the reconstructed 2D map in figure $12 \mathrm{~b}$ ). In addition to this, the light collection is probably less performing in case of photons interacting on the block borders, causing the loss of events. By comparing the two maps, an overall reduced efficiency for the detection of photons beyond $1 \mathrm{MeV}$ 


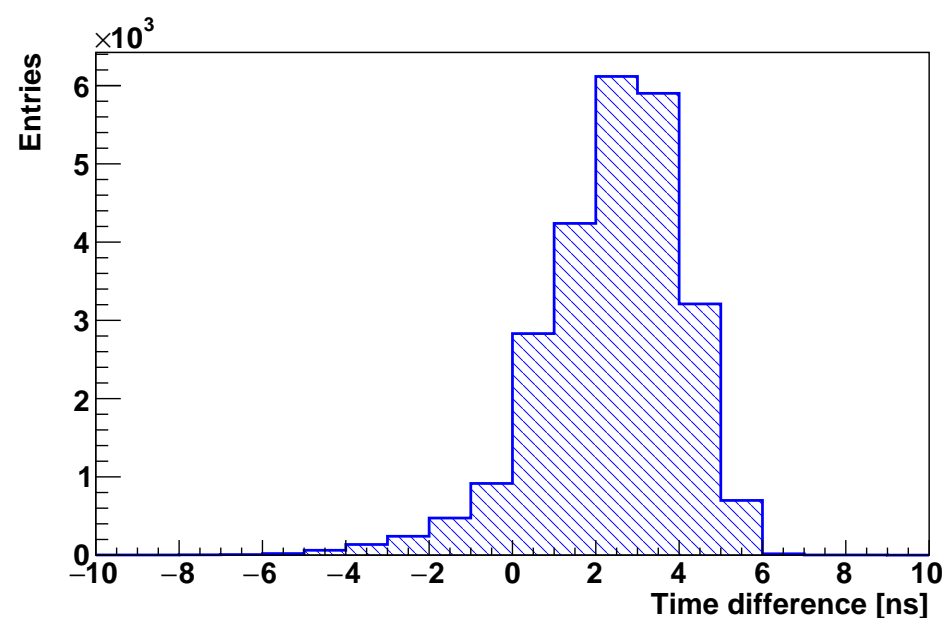

Figure 17: Distribution of arrival time differences between reference scintillator and BGO block.

is verified. This is expected given the factor $\sim 2$ difference in the photopeak efficiency of BGO at $511 \mathrm{keV}$ and $1275 \mathrm{keV}$ [16]. Moreover, the reduced efficiency in the central block area for $1275 \mathrm{keV}$ photons can be related to the photo-multiplier tubes round geometry, which determines a dead space in the center of the block back surface (see [17]). The effect of the dead area is diminished by the light sharing effect at low energy, but it becomes considerable in case of deep-penetrating higher energy photons. In order to fully understand the relative and absolute efficiency of each block section, an irradiation with a point-like collimated source scanning the whole active area is foreseen. This will allow one to precisely define the detection rate variations on the block active area.

\subsection{Time characterization}

Figure 17 shows the distribution of arrival time differences between the reference $\mathrm{BaF}_{2}$ detector and the tested BGO block. The time resolution is defined as the FWHM of this distribution.

\subsection{Results for a set of blocks}

In table 2 the results obtained for the calibration and characterization of a set of ten blocks are listed. The set of studied blocks, corresponding to one third of the complete gamma cameras absorber, shows very uniform results, with an average energy resolution of 25\% FWHM at $511 \mathrm{keV}$ and $20 \%$ FWHM at $1275 \mathrm{keV}$ and an average time resolution of $4.4 \mathrm{~ns}$ FWHM tested with coincidences of $511 \mathrm{keV}$ photons. Both the energy and time resolution are expected to be improved for the detection of photons in the prompt-gamma energy range, in particular above $1 \mathrm{MeV}$.

\section{Discussion and conclusions}

The CLaRyS collaboration is developing in parallel two gamma cameras for ion beam therapy monitoring and nuclear medicine applications. At present, the detector components are being tested separately for characterization purposes. We described here the method developed to calibrate and 
Table 2: Calibration and characterization results for 10 tested BGO blocks.

\begin{tabular}{cccc}
\hline $\begin{array}{c}\text { BGO block } \\
\text { ID }\end{array}$ & $\begin{array}{c}\text { Energy resolution } \\
\text { @ 511 keV } \\
\text { FWHM [\%] }\end{array}$ & $\begin{array}{c}\text { Energy resolution } \\
\text { @ 1275 keV } \\
\text { FWHM [\%] }\end{array}$ & $\begin{array}{c}\text { Time } \\
\text { resolution } \\
\text { FWHM [ns] }\end{array}$ \\
\hline $\begin{array}{c}\text { Ref. block } \\
\mathbf{7 6 2 7}\end{array}$ & $\mathbf{2 3}$ & $\mathbf{1 8}$ & \\
\hline 3166 & 27 & 24 & $\mathbf{4 . 0}$ \\
3184 & 24 & 20 & 4.4 \\
4368 & 25 & 20 & 4.3 \\
7258 & 26 & 19 & 5.3 \\
7581 & 26 & 23 & 4.9 \\
7601 & 24 & 19 & 4.1 \\
7612 & 23 & 19 & 4.1 \\
7651 & 24 & 20 & 4.1 \\
14676 & 25 & 19 & 3.9 \\
\hline Complete set & $25 \pm 1$ & $20 \pm 2$ & 5.1 \\
\hline
\end{tabular}

characterize the response of the bismuth germanate blocks composing the absorber of the two cameras, and we presented the results for a reference set of blocks. The test method has been inspired by previous works, in particular by the work reported in [10]. In addition to the PMT gain equilibration and to the pixel-based energy calibration, we added here an automatic algorithm for the pseudo-pixel position identification and an improved method to assign each interaction to the proper pseudo-pixel. This algorithm also allowed us to analyze the efficiency of the studied blocks in different zones. Finally, we estimated the time resolution of the studied blocks.

The retrieved energy and time resolutions are in agreement with the design expectations; they have been extracted here with relatively low energy photons, and they are expected to improve for the detection of photons in the prompt-gamma energy range. The detection efficiency is generally lower on the block borders, in particular in the four corners; the expected efficiency reduction with increasing incident photon energy is also verified. It must be noticed that the tested blocks have been produced and optimized for PET applications, and so to detect $511 \mathrm{keV}$ photons. The obtained results also verify the possibility to apply these blocks for the detection of higher energy photons. Concerning the spatial reconstruction accuracy, the performed collimated irradiations confirmed the limitation to the pseudo-pixel size; a sub-pixel resolution seems not achievable. In the complete cameras, each event will be assigned to the center of the most-likely pixel, retrieved with the automatic method developed here.

All the tests shown here have been performed with a temporary acquisition system, while the final acquisition of the camera is still under test for optimization. The calibration factors used in this phase can be easily adapted to the final acquisition, which makes use of the same pre-amplification cards described in 2 and shown in figure 1d.

As mentioned, the developed automatic algorithm for spatial reconstruction will be used for 
the single interaction reconstruction in the final configuration; the needed calculation time will be tested for the application in online monitoring, for which a fast reconstruction is required.

The whole set of BGO blocks composing the absorber detector will be characterized with this method, and the whole system will be tested on beam in the next future after verification of the acquisition chain.

\section{Acknowledgments}

This work was supported by the LABEX PRIMES (ANR-11-LABX-0063) of Universite de Lyon and by France HADRON (ANR-11-INBS-0007), within the program "Investissements d'Avenir" (ANR-11-IDEX-0007) operated by the French National Research Agency (ANR).

Moreover, this work has been carried out within the MediNet network, in the ENSAR2 activity. Both projects have received funding from the European Union's Horizon 2020 research and innovation programme grant agreement No 654002 .

\section{References}

[1] Knopf A.C, Lomax A., In vivo proton range verification: a review, Physics in Medicine and Biology 15 (2013) pg.R131-R160

[2] Krimmer, J. and Dauvergne, D. and Létang, J.M. and Testa, É, Prompt-gamma monitoring in hadrontherapy: A review, Nuclear Instruments and Methods in Physics Research Section A: Accelerators, Spectrometers, Detectors and Associated Equipment (2018) pg. 58-73

[3] Bragg, W. H. M. A. and Kleeman, R., On the ionization curves of radium, The London, Edinburgh, and Dublin Philosophical Magazine and Journal of Science 8 (1904) pg.726-738

[4] Min, C.-H. and Kim, C. H. and Youn, M.-Y. and Kim, J.-W., Prompt gamma measurements for locating the dose falloff region in the proton therapy, Applied Physics Letters 18 (2006) pg.183517

[5] Testa, É and Bajard, M. and Chevallier, M. and Dauvergne, D. and Le Foulher, F. and Freud, N. and LÃltang, J.-M. and Poizat, J.-C. and Ray, C. and Testa, M., Monitoring the Bragg peak location of 73 MeVấT Ţu carbon ions by means of prompt $\gamma$-ray measurements, Applied Physics Letters 9 (2008) pg.093506

[6] Krimmer, J. and Ley, J.-L. and Abellan, C. and Cachemiche, J.-P. and Caponetto, L. and Chen, X. and Dahoumane, M. and Dauvergne, D. and Freud, N. and Joly, B. and Lambert, D. and Lestand, L. and Létang, J. M. and Magne, M. and Mathez, H. and Maxim, V. and Montarou, G. and Morel, C. and Pinto, M. and Ray, C. and Reithinger, V. and Testa, É and Zoccarato, Y., Development of a Compton camera for medical applications based on silicon strip and scintillation detectors, Nuclear Instruments and Methods in Physics Research Section A: Accelerators, Spectrometers, Detectors and Associated Equipment 787 (2015) pg.98-101

[7] Casey, M. E. and Nutt, R., A Multicrystal Two Dimensional BGO Detector System for Positron Emission Tomography, IEEE Transactions on Nuclear Science 33 (1986) pg. 460-463

[8] Rogers, J. G. and Nutt, R. and Andreaco, M. and Williams, C. W., Testing 144- and 256-crystal BGO block detectors, IEEE Transactions on Nuclear Science 41 (1994) pg. 1423-1429

[9] Tornai, M. P. and Germano, G. and Hoffman, E. J., Positioning and energy response of PET block detectors with different light sharing schemes, IEEE Transactions on Nuclear Science 41 (1994) pg. $1458-1463$ 
[10] Golnik, C., Treatment verification in proton therapy based on the detection of prompt gamma-rays, $\mathrm{PhD}$ thesis - TU Dresden and Oncoray (2015) pg. 56-59

[11] Elftmann, R. and Tammen, J. and Kulkarni, S. R. and Martin, C. and Böttcher, S. and Wimmer-Schweingruber, R. Characterization of an LSO scintillator for space applications, Journal of Physics: Conference Series 632 (2015)

[12] Brunner, S. E. and Schaart, D. R., BGO as a hybrid scintillator / Cherenkov radiator for cost-effective time-of-flight PET, Physics in medicine and biology, (2017) pg. 4421

[13] Ortega, P. G. and Torres-Espallardo, I. and Cerutti, F. and Ferrari, A. and Gillam, J. E. and Lacasta, C. and Llosa, G. and Oliver, J. F. and Sala, P. R. and Solevi, P. and Rafecas, M., Noise evaluation of Compton camera imaging for proton therapy., Physics in medicine and biology (2015) pg. 1845-1863

[14] Breton, D. and Delagnes, E. and Maalmi, J. and Rusquart, P., The WaveCatcher family of SCA-based 12-bit 3.2-GS/s fast digitizers, 2014 19th IEEE-NPSS Real Time Conference (2014) pg. 1-8

[15] Brun, R. and Rademakers, F., ROOT - An Object Oriented Data Analysis Framework, Proceedings AIHENP'96 Workshop, Lausanne, Sep. 1996, Nucl. Inst. \& Meth. in Phys. Res. A 389 (1997), pg. $81-86$

[16] Saint-Gobain, BGO Bismuth Germanate Scintillation Material, Material datasheet, (2016)

[17] Uribe, J. and Li, H. and Xing, T. and Liu, Y. and Baghaei, H. and Wang, Y. and Aykac, M. and Ramirez, R. and Wong, W.-H., Signal characteristics of individual crystals in high resolution BGO detector designs using PMT-quadrant sharing, IEEE Transactions on Nuclear Science, (2003), pg. $355-361$ 